\title{
Bandwidth Allocation in Ad Hoc Networks: A Price-Based Approach
}

\author{
Ying Qiu \\ Department of Computer Science \\ University of Toronto \\ Toronto, ON, M5S 3G4 \\ Canada \\ Email: yingq@cs.toronto.edu
}

\author{
Peter Marbach \\ Department of Computer Science \\ University of Toronto \\ Toronto, ON, M5S 3G4 \\ Canada \\ Email: marbach@cs.toronto.edu
}

\begin{abstract}
We consider pricing as a means to stimulate cooperation in ad hoc networks: users can charge other users a price for relaying their data packets. Assuming that users set prices to maximize their own net benefit, we propose an iterative price and rate adaption algorithm. We show that this algorithm converges to a socially optimal bandwidth allocation. We use a numerical case study to illustrate our results.
\end{abstract}

\section{INTRODUCTION}

An ad hoc network is a collection of wireless nodes (users) which form a network without relying on any existing network infrastructure or centralized administration. Ad hoc networks have been proposed for situations where it is not practical or affordable to set up fixed network infrastructure. Due to the infrastructure-less nature of ad hoc networks, all network functions have to be performed by the participating users. For example, when a node wants to send data packets to a destination node which is outside its transmission range, then other users in the network have to relay the packets to the destination. However, users with limited bandwidth and battery resources might be reluctant to forward data packets for other users, unless there is an additional mechanism in place to give users an incentive to provide this service. In this paper, we consider pricing as a means to stimulate cooperation in an ad hoc network. In particular, we assume that users can charge other users for forwarding their data packets. The aim of the paper is to study how users set their prices for forwarding packets, and how much bandwidth they allocate for relaying data packets for other users.

When bandwidth and battery resources are scarce in an ad hoc network, then users might not volunteer to forward packets for other users as this impacts the ability to transmit their own traffic. To model this situation, we associate with each user a cost which consists of the following three components: bandwidth, battery cost, and interference cost. When a user has limited transmission resources, then relaying packets for other users will incur a bandwidth cost as it reduces the bandwidth available to the user to transmit its own traffic. Relaying packets for other users will also drain the battery and incur a battery cost. Finally, interference between users who transmit at the same time instant can cause bit errors and packet loss. In the case of loss data packets have to be retransmitted, thus further draining the batteries and incurring an additional interference cost. In our analysis, we will focus on the bandwidth cost; in Section VIII, we briefly indicate how our model can be extended to incorporate battery and interference cost.

Providing users an incentive to relay packets is an important problem in ad hoc networks where users are independent entities and do not voluntarily relay packets for other users. However, this problem has not received much attention; to our knowledge it has only been investigated as part of a research project on wireless networks formed by "terminodes" (see [1]). Terminodes are personal devices that provide the functionality of both a terminal (end host) and a network node (router). In [2], Buttyán and Hubaux use a virtual currency (called nuglets) as a means to provide terminodes an incentive to relay packets for other terminodes: terminodes must need to have a certain amount of nuglets to send traffic, and nuglets can only be acquired by forwarding packets for other users. The assumption that terminodes can only acquire nuglets by forwarding packets for other nodes introduces a budget constraint.

The use of pricing as a means for allocating resources in communication networks has received much attention in recent years. In particular, the work by Kelly et al. ([3]) for wired networks spurred a plethora of research interests. In their work, Kelly et al. propose a scheme where a network provider charges users as a function of the traffic load on the individual links in the network, and users accessing the network decide on their transmission rate as a function of these network prices. Kelly et al. show that this pricing scheme can be used to achieve (in equilibrium) a weighted proportional fair rate allocation. The pricing mechanism for ad hoc networks that we consider reduces to the pricing scheme in [3] for the special case where each user in the network either sends only its own traffic (acts as an end host), or only relays traffic for other users (acts as a router). We will comment on this in more details in Section V.

Although the problem considered here is motivated by the bandwidth allocation problem in ad hoc networks, we believe that the price-based resource allocation scheme that we present can be applied to any "peer-to-peer systems" with 
the characteristic that independent peers own a resource which can either be used by the peers themselves, or shared with other peers. In the context that we consider here, the users' resources are given in terms of their transmission capacity; in peer-to-peer computing systems, the resources would be given in terms of available CPU cycles at each node (peer); in peer-to-peer content delivery networks, the resources would be given in terms of the cache space available at individual nodes.

The rest of the paper is organized as follows. In Section II, we define a priced-based bandwidth allocation mechanism. In Section III, we study an optimal allocation by an individual user. In Section IV we propose an iterative price and bandwidth adaptation algorithm. In Section V, we briefly review the work by Kelly et al. In Section VI, we analyze the convergence behavior of the iterative algorithm and show that it converges to a unique bandwidth allocation which maximizes the social welfare of the network. Section VII illustrates the numerical results in the simulation of the algorithm. In Section VIII, we briefly outline how our model can be extended to incorporate battery lifetime constraints and user interference. Due to space constraints, we state most results without proofs; detailed derivations can be found in [5].

\section{PRICING Model}

In this section, we describe the pricing model that we consider. For the analysis, we assume a static network topology, where each user sends traffic to a single destination node along a single and fixed path.

Consider an ad hoc network which consists of a set $\mathcal{N}$ of users given by $\mathcal{N}=\{1,2, \cdots, N\}$. We assume that each user $n \in \mathcal{N}$ sends traffic along a single, fixed path $r_{n}$ where $r_{n}$ indicates the set of nodes that relay traffic for user $n$ (excluding user $n$ ). Let $G(n)$ be set of users for which user $n$ relays traffic (excluding user $n$ ), and let $H(n)=G(n) \cup\{n\}$. Note that $H(n)$ is the set of all users for which user $n$ sends and relays traffic (including user $n$ ). Let $A=\left(A_{n m}, n, m \in \mathcal{N}\right.$ ) be the routing matrix given by

$$
A_{n m}= \begin{cases}1 & \text { if } n \in H(m) \\ 0 & \text { otherwise. }\end{cases}
$$

We associate user $n$ the transmission capacity $C_{n}$. Let $x_{n} \geq$ 0 be the transmission rate that user $n$ allocates for sending its own traffic, and let $y_{n} \geq 0$ be the rate allocated for relaying traffic for other users. Naturally, we have the constraint that $x_{n}+y_{n} \leq C_{n}$. In addition, we assume that each user $n \in \mathcal{N}$ charges other users a price $\mu_{n}$ (per unit flow) for forwarding their traffic. The aggregated price (cost) $\lambda_{n}$ that user $n$ has to pay other users along its route for relaying its traffic is then given by

$$
\lambda_{n}=\sum_{m \in r_{n}} \mu_{m} .
$$

In the following, we model how user $n$ decides on

(a) $x_{n}$, the transmission rate for sending its own traffic,

(b) $y_{n}$, the transmission rate allocated to relay traffic of other users,

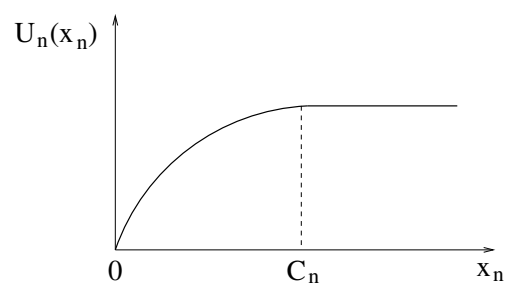

Fig. 1. Utility function $U_{n}\left(x_{n}\right)$ of user $n$.

(c) $\mu_{n}$, the price user $n$ charges for relaying traffic.

\section{A. User Utility Function}

We associate with each user $n \in \mathcal{N}$ a utility function $U_{n}\left(x_{n}\right)$ which depends on the transmission rate $x_{n}$ that user $n$ allocates for sending its own traffic [4]. We make the following assumption (see Figure 1 for an illustration).

Assumption 1: For each user $n \in \mathcal{N}$, the utility function $U_{n}: \Re_{+} \rightarrow \Re_{+}$is twice continuously differentiable and has a bounded derivative $U_{n}^{\prime}\left(x_{n}\right)$. Furthermore, $U_{n}\left(x_{n}\right)$ is strictly concave on $\left[0, C_{n}\right]$ and we have

$$
U_{n}(0)=0
$$

and

$$
U_{n}\left(x_{n}\right)=U_{n}\left(C_{n}\right), \quad \text { for } x_{n} \geq C_{n} .
$$

We note that utility functions with these characteristics are commonly used in the pricing literature (see for example [3]). The assumption that $U_{n}\left(x_{n}\right)=U_{n}\left(C_{n}\right)$, for $x_{n} \geq C_{n}$ reflects that capacity constraint that $x_{n} \leq C_{n}$. Assumption 1 does not require that all users have the same utility function. Throughout the paper, we assume that the utility function $U_{n}\left(x_{n}\right)$ is private information known to user $n$, but not to other users.

\section{B. User Demand Function}

We associate with user $n$ the demand function $D_{n}\left(\lambda_{n}\right)$, where $D_{n}\left(\lambda_{n}\right)$ is the optimal solution to the following maximization problem,

$$
D_{n}\left(\lambda_{n}\right)=\arg \max _{0 \leq x_{n} \leq C_{n}}\left\{U_{n}\left(x_{n}\right)-\lambda_{n} x_{n}\right\}, \quad \lambda_{n} \geq 0 .
$$

The value $D_{n}\left(\lambda_{n}\right)$ is equal to the transmission rate $x_{n}$ that maximizes the net benefit of user $n$ given that user $n$ does not need to forward traffic for other users, and the cost per unit flow is equal to $\lambda_{n}$. Assumption 1 implies that $D_{n}(\cdot)$ is given as follows.

$$
D_{n}\left(\lambda_{n}\right)= \begin{cases}C_{n} & \lambda_{n}=0 \\ U_{n}^{\prime-1}\left(\lambda_{n}\right) & 0<\lambda_{n}<U_{n}^{\prime}(0) \\ 0 & \lambda_{n} \geq U_{n}^{\prime}(0)\end{cases}
$$

Note that $D_{n}\left(\lambda_{n}\right)$ is bounded by $C_{n}$. Furthermore, we have the following lemma.

Lemma 1: The demand function $D_{n}\left(\lambda_{n}\right)$ is continuous and has a bounded first derivative $D_{n}^{\prime}\left(\lambda_{n}\right)$. In addition, there exists a constant $B_{n}>0$ such that

$$
D_{n}^{\prime}\left(\lambda_{n}\right) \geq B_{n}, \quad \text { for } 0 \leq \lambda_{n} \leq U_{n}^{\prime}(0) .
$$


The above lemma follows immediately from Assumption 1 and we omit a detailed proof here. Note that Lemma 1 implies that the demand function $D_{n}\left(\lambda_{n}\right)$ is strictly decreasing on $\left[0, U_{n}^{\prime}(0)\right]$.

\section{External Demand Function}

We call the amount of traffic to be relayed at user $n$ the external demand at user $n$. The external demand depends on the price $\mu_{n}$ that node $n$ charges for its relay service, as well as on the prices at other nodes. For example, when user $n$ charges a low price $\mu_{n}$, but all other nodes charge a high price for their relay service, then the external demand at node $n$ might still be low, and vice versa.

Let $I_{n}\left(\mu_{n}, \mu_{-n}\right)$ denote the external demand at user $n$, where $\mu_{-n} \in \Re_{+}^{N-1}$ is the vector listing the prices set by all users other than $n$. Note that $I_{n}\left(\mu_{n}, \mu_{-n}\right)$ is upper-bounded by $\sum_{m \in G(n)} D_{m}\left(\lambda_{m}\right)$, the total demand under the idealized situation when only user $n$ relays traffic and no loss occurs in the network. We have the following result.

Lemma 2: For every price vector $\mu_{-n} \in \Re_{+}^{N-1}$, the external demand function $I_{n}\left(\mu_{n}, \mu_{-n}\right)$ is decreasing and continuous in $\mu_{n}$. Furthermore, for every price vector $\mu_{-n} \in \Re_{+}^{N-1}$, $I_{n}\left(\mu_{n}, \mu_{-n}\right)$ is differentiable with respect to $\mu_{n}$ except at a finite set of points $\mu_{n} \in \Re_{+}$.

Lemma 2 implies that the right derivative of $I_{n}\left(\mu_{n}, \mu_{-n}\right)$ with respect to $\mu_{n}$ exists.

At a price $\mu_{n}$ such that $I_{n}\left(\mu_{n}, \mu_{-n}\right)>0$, we define the elasticity $l_{n}\left(\mu_{n}, \mu_{-n}\right)$ of the external demand by

$$
l_{n}\left(\mu_{n}, \mu_{-n}\right)=\left|\frac{I_{n}\left(\mu_{n}, \mu_{-n}\right)}{d_{n}^{+}\left(\mu_{n}, \mu_{-n}\right)}\right|,
$$

where $d_{n}^{+}\left(\mu_{n}, \mu_{-n}\right)$ is the right derivative of $I_{n}\left(\mu_{n}, \mu_{-n}\right)$ with respect to $\mu_{n}$. We say that the external demand at $\mu_{n}$ is elastic when there exists a constant $L_{n}$ such that $l_{n}\left(\mu_{n}, \mu_{-n}\right) \leq L_{n}$ for every $\mu_{n} \in \Re_{+}$and $\mu_{-n} \in \Re_{+}^{N-1}$ such that $I_{n}\left(\mu_{n}, \mu_{-n}\right)>$ 0 . When the external demand is elastic, then a small change in the price $\mu_{n}$ can cause a large change in the external demand with respect to the current demand $I_{n}\left(\mu_{n}, \mu_{-n}\right)$. Throughout the paper, we assume that the external demand is elastic. In particular, we consider the limiting case where $L_{n} \rightarrow 0$.

\section{User Optimization Problem}

Given a vector $\mu_{-n} \in \Re_{+}^{N-1}$ indicating the prices of all users except user $n$, we associate with user $n$ the net benefit

$$
U_{n}\left(x_{n}\right)-x_{n} \lambda_{n}+y_{n} \mu_{n}
$$

where $U_{n}\left(x_{n}\right)$ is the utility associated with rate $x_{n}, x_{n} \lambda_{n}$ is cost for sending its own traffic, and $y_{n} \mu_{n}$ is the income from relaying traffic for other users. We then assume that each user chooses an allocation to maximize its net benefit. This objective is captured by the maximization problem

$$
\begin{array}{cc}
\operatorname{USER}\left(U_{n}, \mu_{-n}\right): & \\
\max _{x_{n}, y_{n}, \mu_{n}} & U_{n}\left(x_{n}\right)-x_{n} \lambda_{n}+y_{n} \mu_{n}, \\
\text { subject to } & x_{n}+y_{n} \leq C_{n}, \\
& y_{n} \leq I_{n}\left(\mu_{n}, \mu_{-n}\right), \\
& x_{n}, y_{n}, \mu_{n} \geq 0,
\end{array}
$$

where $\lambda_{n}=\sum_{m \in r_{n}} \mu_{m}$. Note that the bandwidth $y_{n}$ that user $n$ allocates for relaying traffic is always less, or equal, to the external demand, i.e., we have that $y_{n} \leq I_{n}\left(\mu_{n}, \mu_{-n}\right)$.

Note that users are not able to directly compute an optimal solution to maximization problem $U S E R\left(U_{n}, \mu_{-n}\right)$, as this requires the knowledge of the external demand function $I_{n}\left(\mu_{n}, \mu_{-n}\right)$; this is an unrealistic assumption for the situation that we consider here. Alternatively, users could update their allocations based on past observations of the system, and iteratively approach an optimal solution. Here, we consider an iterative algorithm where users choose at each iteration first choose an optimal bandwidth allocation based on the current prices, and then update the price they charge for relaying traffic.

More precisely, we assume that at the beginning of iteration step $k(k \in \mathbb{N})$, each user $n$ has available from the previous iteration step $k-1$ the prices $\mu_{n}^{k-1}$ and $\lambda_{n}^{k-1}$, as well as the external demand $i_{n}^{k-1}=I_{n}\left(\mu_{n}^{k-1}, \mu_{-n}^{k-1}\right)$. Based on these observations, user $n$ chooses then an allocation $\left(x_{n}^{k}, y_{n}^{k}, \mu_{n}^{k}\right)$ as follows.

First, user $n$ keeps its price $\mu_{n}^{k-1}$ fixed and chooses a bandwidth allocation $\left(x_{n}^{k}, y_{n}^{k}\right)$ such that

$$
\max _{x_{n}, y_{n}}\left\{U_{n}\left(x_{n}\right)-x_{n} \lambda_{n}^{k-1}+y_{n} \mu_{n}^{k-1}\right\}
$$

subject to

$$
\begin{gathered}
x_{n}+y_{n} \leq C_{n} \\
y_{n} \leq i_{n}^{k-1} \\
x_{n}, y_{n} \geq 0
\end{gathered}
$$

Note that this maximization problem is similar to (1), however, now the external demand function $I_{n}\left(\mu_{n}, \mu_{-n}^{k-1}\right)$ is replaced by the value $i_{n}^{k-1}=I_{n}\left(\mu_{n}^{k-1}, \mu_{-n}^{k-1}\right)$ of the observed external demand in time slot $k-1$ under the price $\mu_{n}^{k-1}$. The following result states that there exists a unique optimal allocation $\left(x_{n}^{k}, y_{n}^{k}\right)$ to the above bandwidth allocation problem.

Lemma 3: There exists a unique allocation $\left(x_{n}^{k}, y_{n}^{k}\right)$ that maximizes the optimization problem given by (2).

Once user $n$ has decided on $\left(x_{n}^{k}, y_{n}^{k}\right)$, user $n$ updates its price by setting

$$
\mu_{n}^{k}=\left[\mu_{n}^{k-1}+\alpha_{n}\left(D_{n}\left(\mu_{n}^{k-1}+\lambda_{n}^{k-1}\right)+i_{n}^{k-1}-C_{n}\right)\right]^{+},
$$

where $\alpha_{n}>0$ is a small step size parameter and $[x]^{+}=$ $\max \{x, 0\}, x \in \Re$. We will derive in Section IV this update rule and show that it indeed increases the net benefit of user $n$. For now, we point out that this update rule has roughly the following interpretation. When price $\mu_{n}^{k-1}$ is too low such 
that the total demand exceeds the transmission capacity $C_{n}$ (i.e., when $\left.D_{n}\left(\mu_{n}^{k-1}+\lambda_{n}^{k-1}\right)+i_{n}^{k-1}>C_{n}\right)$ ), then user $n$ increases the price by a little bit to reduce demand. When price $\mu_{n}^{k-1}$ is too high and user $n$ has spare capacity (i.e., when $\left.D_{n}\left(\mu_{n}^{k-1}+\lambda_{n}^{k-1}\right)+i_{n}^{k-1}<C_{n}\right)$, then user $n$ decreases the price by a little bit to stimulate demand.

\section{E. Results}

In the following, we analyze the iterative allocation algorithm described above. In our main result, we show that under this algorithm each user converges to an allocation which maximizes its own net benefit. Furthermore, the bandwidth allocation in equilibrium is unique and maximizes the sum of the utilities of all users.

This result implies that it is indeed in the users' interest to adopt this algorithm as it leads to an allocation which maximizes their individual net benefit. In addition, the result suggests that using pricing to stimulate cooperation will lead to good network performance, as in equilibrium the network bandwidth is shared such that the sum of the utilities of all users is maximized.

\section{Optimal Solution}

In this section, we derive the necessary and sufficient conditions for an optimal solution $\left(x_{n}^{*}, y_{n}^{*}, \mu_{n}^{*}\right)$ to the maximization problem $U S E R\left(U_{n}, \mu_{-n}\right)$ given by (1).

Consider a fixed user $n$. Furthermore, let $\mu_{-n} \in \Re_{+}^{N-1}$ be a given price vector indicating the prices of all users except user $n$, and let $\lambda_{n}=\sum_{m \in r_{n}} \mu_{m}$ be the corresponding aggregated price that user $n$ has to pay to forward its traffic. Assume that all users other than user $n$ keep their prices fixed (i.e., $\mu_{-n}$ and $\lambda_{n}$ are fixed), but adapt their bandwidth allocation depending on the price $\mu_{n}$ set by user $n$. In this case, the external demand at node $n$ depends only on the price $\mu_{n}$, and we use $I_{n}\left(\mu_{n}\right)$, instead of $I_{n}\left(\mu_{n}, \mu_{-n}\right)$, to denote the external demand at node $n$.

For the non-trivial case where $I_{n}(0)>0$, we obtain the following results (otherwise the problem reduces to the optimization problem given in Subsection II-B).

Lemma 4: An allocation $\left(x_{n}^{*}, y_{n}^{*}, \mu_{n}^{*}\right)$ such that $x_{n}^{*}+y_{n}^{*}=$ $C_{n}$, is an optimal solution of $U S E R\left(U_{n}, \mu_{-n}\right)$ if and only if

$$
x_{n}^{*}=D_{n}\left(\mu_{n}^{*}+\lambda_{n}\right)
$$

and

$$
y_{n}^{*}=I_{n}\left(\mu_{n}^{*}\right) .
$$

Note that the equality $y_{n}^{*}=I_{n}\left(\mu_{n}^{*}\right)$ implies that there is no traffic loss at user $n$.

Proof: When $x_{n}^{*}+y_{n}^{*}=C_{n}$, we have that

$$
\begin{aligned}
R_{n}\left(x_{n}^{*}, y_{n}^{*}, \mu_{n}^{*}\right) & =U_{n}\left(x_{n}^{*}\right)-x_{n}^{*} \lambda_{n}+y_{n} \mu_{n}^{*} \\
& =U_{n}\left(x_{n}^{*}\right)-x_{n}^{*}\left(\mu_{n}^{*}+\lambda_{n}\right)+C_{n} \mu_{n}^{*} .
\end{aligned}
$$

The first order condition then implies that $x_{n}^{*}=D_{n}\left(\mu_{n}^{*}+\lambda_{n}\right)$.
Next, suppose that $y_{n}^{*}<I_{n}\left(\mu_{n}^{*}\right)$. Since $I_{n}\left(\mu_{n}^{*}\right)$ is continuous and decreasing, there exists a price $\hat{\mu_{n}}$ such that $\hat{\mu_{n}}>\mu_{n}^{*}$ and $y_{n}^{*}<I_{n}\left(\hat{\mu_{n}}\right) \leq I_{n}\left(\mu_{n}^{*}\right)$. Note that

$$
R_{n}\left(x_{n}^{*}, y_{n}^{*}, \hat{\mu_{n}}\right)-R_{n}\left(x_{n}^{*}, y_{n}^{*}, \mu_{n}^{*}\right)=y_{n}^{*}\left(\hat{\mu_{n}}-\mu_{n}^{*}\right)>0 \text {, }
$$

which contradicts the fact that $\left(x_{n}^{*}, y_{n}^{*}, \mu_{n}^{*}\right)$ is an optimal solution, and it follows that $y_{n}^{*}=I_{n}\left(\mu_{n}^{*}\right)$.

Lemma 5: An allocation $\left(x_{n}^{*}, y_{n}^{*}, \mu_{n}^{*}\right)$, such that $x_{n}^{*}+y_{n}^{*}<$ $C_{n}$, is an optimal solution of $\operatorname{USER}\left(U_{n}, \mu_{-n}\right)$ if and only if

$$
\begin{aligned}
x_{n}^{*} & =D_{n}\left(\lambda_{n}\right), \\
y_{n}^{*} & =I_{n}\left(\mu_{n}^{*}\right), \\
\mu_{n}^{*} & =\arg \max _{\mu_{n} \geq 0}\left\{\mu_{n} I\left(\mu_{n}\right)\right\} .
\end{aligned}
$$

Furthermore, when there exists a constant $L_{n}>0$ such that $l_{n}\left(\mu_{n}, \mu_{-n}\right) \leq L_{n}$ for all $\mu_{n} \in \Re_{+}$at which $I_{n}\left(\mu_{n}, \mu_{-n}\right)>$ 0 , then we have that $\mu_{n}^{*} \leq L_{n}$.

Proof: When $x_{n}^{*}+y_{n}^{*}<C_{n}$, then the net benefit of user $n$ is equal to

$$
U_{n}\left(x_{n}\right)-x_{n} \lambda_{n}+y_{n} \mu_{n} .
$$

From the first order condition, it follows that $x_{n}^{*}=D_{n}\left(\lambda_{n}\right)$. By the same argument as given for Lemma 4, we have that $y_{n}^{*}=I_{n}\left(\mu_{n}^{*}\right)$.

Furthermore, the optimal price $\mu_{n}^{*}$ satisfies

$$
\mu_{n}^{*}=\arg \max _{\mu_{n} \in \mathbb{R}^{+}}\left\{\mu_{n} I_{n}\left(\mu_{n}\right)\right\} .
$$

Differentiating $\mu_{n} I_{n}\left(\mu_{n}\right)$ with respect to $\mu_{n}$, we obtain

$$
\left(\mu_{n} I_{n}\left(\mu_{n}\right)\right)^{\prime}=D_{n}^{\prime}\left(\mu_{n}\right)\left(\mu_{n}+\frac{I_{n}\left(\mu_{n}\right)}{I_{n}^{\prime}\left(\mu_{n}\right)}\right) .
$$

It follows that when $\mu_{n}^{*}$ is optimal, then we have that $\left(\mu_{n}^{*} I_{n}\left(\mu_{n}^{*}\right)\right)^{\prime} \leq 0$, which implies that $\mu_{n}^{*} \leq L_{n}$.

Combining Lemma 4 and 5, we obtain for the limiting case where $L_{n} \rightarrow 0$, that the allocation $\left(x_{n}^{*}, y_{n}^{*}, \mu_{n}^{*}\right)$ is an optimal allocation if and only if

$$
\begin{aligned}
x_{n}^{*} & =D_{n}\left(\mu_{n}^{*}+\lambda_{n}\right) \\
y_{n}^{*} & =I_{n}\left(\mu_{n}^{*}\right) \\
\mu_{n}^{*} & =0, \quad \text { if } x_{n}^{*}+y_{n}^{*}<C_{n} .
\end{aligned}
$$

\section{ITERATIVE ALGORITHM}

In this section, we outline the derivation the iterative algorithm described in Section II. The derivation of the algorithm is quite involved and we refer to [5] for a detailed derivation. Again, we consider the limiting case where $L_{n} \rightarrow 0$, for $n \in \mathcal{N}$.

\section{A. Bandwidth Allocation}

We first consider how user $n$ decides on its bandwidth allocation $\left(x_{n}^{k}, y_{n}^{k}\right)$ at time step $k$. Recall that user $n$ will choose $\left(x_{n}^{k}, y_{n}^{k}\right)$ to maximize the optimization problem given by (2). To compute the optimal solution to (2), we distinguish three different cases. 
When

$$
D_{n}\left(\mu_{n}^{k-1}+\lambda_{n}^{k-1}\right)+i_{n}^{k-1} \geq C_{n},
$$

then it can be shown that the optimal solution $\left(x_{n}^{k}, y_{n}^{k}\right)$ to (2) has the property that

$$
x_{n}^{k}+y_{n}^{k}=C_{n} .
$$

Using a similar argument as in the proof of Lemma 4, it follows that in this case the optimal solution $\left(x_{n}^{k}, y_{n}^{k}\right)$ to (2) given by

$$
\begin{aligned}
& x_{n}^{k}=D_{n}\left(\mu_{n}^{k-1}+\lambda_{n}^{k-1}\right) \\
& y_{n}^{k}=C_{n}-x_{n}^{k} .
\end{aligned}
$$

When we have that

$$
D_{n}\left(\lambda_{n}^{k-1}\right)+i_{n}^{k-1}<C_{n},
$$

then it can be shown that the optimal solution $\left(x_{n}^{k}, y_{n}^{k}\right)$ to (2) has the property that

$$
x_{n}^{k}+y_{n}^{k}<C_{n} .
$$

In this case, a similar argument as used in the proof of Lemma 5 can be used to show that the optimal solution $\left(x_{n}^{k}, y_{n}^{k}\right)$ is given by

$$
\begin{aligned}
& x_{n}^{k}=D_{n}\left(\lambda_{n}^{k-1}\right) \\
& y_{n}^{k}=i_{n}^{k-1} .
\end{aligned}
$$

Finally, we consider the case when

$$
D_{n}\left(\mu_{n}^{k-1}+\lambda_{n}^{k-1}\right)+i_{n}^{k-1}<C_{n}
$$

and

$$
D_{n}\left(\lambda_{n}^{k-1}\right)+i_{n}^{k-1} \geq C_{n} .
$$

In this case, one can show that the optimal solution to the maximization problem (2) is then given by

$$
\begin{aligned}
& x_{n}^{k}=C_{n}-i_{n}^{k-1} \\
& y_{n}^{k}=i_{n}^{k-1} .
\end{aligned}
$$

We refer to [5] for a detailed derivation.

Figure 2 summarizes the bandwidth allocation by user $n$ at step $k$.

\section{B. Price Update}

Next, we outline the derivation of the price update rule at step $k$.

Lemma 4 implies that when

$$
D_{n}\left(\mu_{n}^{k-1}+\lambda_{n}^{k}\right)+I_{n}\left(\mu_{n}^{k}, \mu_{-n}^{k-1}\right)=C_{n},
$$

then the allocation $\left(x_{n}^{k}, y_{n}^{k}, \mu_{n}^{k-1}\right)$ is optimal and the price $\mu_{n}^{k-1}$ should not be changed.

Lemma 5 implies that when $\mu_{n}^{k-1}$ is equal to 0 and

$$
D_{n}\left(\mu_{n}^{k-1}+\lambda_{n}^{k}\right)+I_{n}\left(\mu_{n}^{k}, \mu_{-n}^{k-1}\right)<C_{n},
$$

then the allocation $\left(x_{n}^{k}, y_{n}^{k}, \mu_{n}^{k-1}\right)$ is optimal and the price $\mu_{n}^{k-1}$ should not be changed.

\section{Bandwidth Allocation:}

If $D_{n}\left(\mu_{n}^{k-1}+\lambda_{n}^{k-1}\right)+i_{n}^{k-1} \geq C_{n}$, then set

$x_{n}^{k}=D_{n}\left(\mu_{n}^{k-1}+\lambda_{n}^{k-1}\right)$

$$
y_{n}^{k}=C_{n}-x_{n}^{k} \text {; }
$$

else if $D_{n}\left(\lambda_{n}^{k-1}\right)+i_{n}^{k-1}<C_{n}$, then set

$$
\begin{aligned}
& x_{n}^{k}=D_{n}\left(\lambda_{n}^{k-1}\right) \\
& y_{n}^{k}=i_{n}^{k-1}
\end{aligned}
$$

else set

$$
\begin{aligned}
& x_{n}^{k}=C_{n}-i_{n}^{k-1} \\
& y_{n}^{k}=i_{n}^{k-1} ;
\end{aligned}
$$

Price Update:

$$
\mu_{n}^{k}=\left[\mu_{n}^{k-1}+\alpha_{n}\left(D_{n}\left(\mu_{n}^{k-1}+\lambda_{n}^{k-1}\right)+i_{n}^{k-1}-C_{n}\right)\right]^{+} .
$$

Fig. 2. Iterative algorithm at user $n$.

Furthermore, it can be shown that when

$$
D_{n}\left(\mu_{n}^{k-1}+\lambda_{n}^{k-1}\right)+I_{n}\left(\mu_{n}^{k-1}, \mu_{-n}^{k-1}\right)>C_{n},
$$

then user $n$ should increase the price $\mu_{n}^{k-1}$ to increase its net benefit; similarly, when

$$
D_{n}\left(\mu_{n}^{k-1}+\lambda_{n}^{k-1}\right)+I_{n}\left(\mu_{n}^{k-1}, \mu_{-n}^{k-1}\right)<C_{n},
$$

then user $n$ should decrease the price $\mu_{n}^{k-1}$ to increase its net benefit.

The above result suggests the following update rule for $\mu_{n}^{k}$,

$$
\mu_{n}^{k}=\left[\mu_{n}^{k-1}+\alpha_{n}\left(D_{n}\left(\mu_{n}^{k-1}+\lambda_{n}^{k-1}\right)+i_{n}^{k-1}-C_{n}\right)\right]^{+},
$$

where $\alpha_{n}>0$ is a small step size parameter.

\section{Price-Based Bandwidth Allocation in Wired POINT-TO-POINT NETWORKS}

Before we analyze the algorithm presented in the previous section, we compare in this section the framework presented above with the one used by Kelly et al. in [3]. In particular, we show that the above model is identical to the one considered by Kelly et al. for the special case where (a) the external demands are very elastic at each user and (b) every user either only generates traffic (acts as an end host) or relays traffic (acts as a router/link). In addition, we use the results by Kelly et al. that we presented in this section for our convergence analysis in the next section.

\section{A. Model}

Kelly et al. consider in [3] the following price-based bandwidth allocation mechanism for wired networks (such as the Internet).

Consider a wired network consisting of a set $J$ of links, and let $C_{j}$ is the finite capacity of link $j$, for $j \in J$. Let $R$ be the set of users accessing the network. Associate with each user $r$ a single route $r$ which is a non-empty subset of $J$. Set $A_{j r}=1$ if $j \in r$, so that the link $j$ lies on route $r$, and set $A_{j r}=0$ otherwise. This defines a 0-1 routing matrix $A=\left(A_{j r}, j \in J, r \in R\right)$.

Suppose that if a rate $x_{r}$ is allocated to user $r$ then this has utility $U_{r}\left(x_{r}\right)$ to the user, where $U_{r}(\cdot)$ satisfies the following assumption. 
Assumption 2: $U_{r}\left(x_{r}\right)$ is increasing, strictly concave and continuously differentiable over the range $x_{r} \geq 0$.

For the framework considered in Section II, the above assumption is equivalent to Assumption 1 as the rate $x_{n}$ at each user $n$ is restricted to the interval $\left[0, C_{n}\right]$.

Let $U=\left(U_{r}(\cdot), r \in R\right)$ and $C=\left(C_{j}, j \in J\right)$, and suppose that the network seeks a rate allocation $x=\left(x_{r}, r \in R\right)$ which solves the following optimization problem.

$S Y \operatorname{STEM}(U, A, C)$

$$
\begin{aligned}
\max & \sum_{r \in R} U_{r}\left(x_{r}\right) \\
\text { subject to } & A x \leq C \\
\text { over } & x \geq 0 .
\end{aligned}
$$

\section{B. Problem Decomposition}

The above maximization problem can not be solved directly by the network provider as it involves utilities $U$ that are unknown by the network. Instead, Kelly et al. consider two simpler problems.

Suppose that user $r$ chooses an amount to pay per unit time $w_{r}$, and receives in return a flow $x_{r}$ given by

$$
x_{r}=\frac{w_{r}}{\lambda_{r}},
$$

where $\lambda_{r}$ is a charge per unit flow for user $r$. Then the utility maximization problem for user $r$ is as follows.

$U S E R\left(U_{r} ; \lambda_{r}\right)$ :

$$
\begin{array}{ll}
\max & U_{r}\left(\frac{w_{r}}{\lambda_{r}}\right)-w_{r} \\
\text { over } & w_{r} \geq 0 .
\end{array}
$$

Note that the above maximization problem is equivalent to the user problem that we defined in Subsection II-D for the case where a user does not relay any traffic, i.e., when the external demand of a user is equal to zero.

Suppose next that the network tries to maximize the function $\sum_{r \in R} w_{r} \log x_{r}$. Setting $w=\left(w_{r}, r \in R\right)$, the network problem is then as follows.

$N E T W O R K(A, C ; w)$ :

$$
\begin{aligned}
\max & \sum_{r \in R} w_{r} \log x_{r} \\
\text { subject to } & A x \leq C \\
\text { over } & x \geq 0 .
\end{aligned}
$$

Note that solving the maximization problem $\operatorname{NETWORK}(A, C ; w)$ does not require the network to know the utilities $U$.

Kelly et al. show that there always exist vectors $\lambda=$ $\left(\lambda_{r}, r \in R\right), w=\left(w_{r}, r \in R\right)$ and $x=\left(x_{r}, r \in R\right)$, satisfying $w_{r}=\lambda_{r} x_{r}$ for $r \in R$, such that $w_{r}$ solves $U S E R\left(U_{r} ; \lambda_{r}\right)$ for $r \in R$ and $x$ solves $N E T W O R K(A, C ; w)$; furthermore, the vector $x$ is the unique solution to $\operatorname{SYSTEM}(U, A, C)$. This result implies that problems $\operatorname{NETWORK}(A, C ; w)$ and $U S E R\left(U_{r} ; \lambda_{r}\right)$ for $r \in R$ can be used to obtain the unique solution to $\operatorname{SYSTEM}(U, A, C)$.
C. Characterizing the Optimal Solution using Lagrange Multipliers

The Lagrangian ([6]) for $\operatorname{NETWORK}(A, C ; w)$ is

$$
L(x, \mu)=\sum_{r \in R} w_{r} \log x_{r}-\mu^{T}(C-A x),
$$

where $\mu=\left(\mu_{j}, j \in J\right)$ is a vector of Lagrange multipliers (link shadow prices).

Let $D_{r}\left(\lambda_{r}\right)$ be the demand of user $r$ when the price per unit flow is $\lambda_{r}$, i.e.,

$$
D_{r}\left(\lambda_{r}\right)=\arg \max _{x_{r} \geq 0}\left\{U_{r}\left(x_{r}\right)-x_{r} \lambda_{r}\right\}, \quad \lambda_{r} \geq 0 .
$$

Furthermore, let $H(j)$ be the set of users whose routes pass link $j$. Kelly et al. then prove the following result for the above Lagrangian.

Proposition 1: The rate vector $x^{*}$ is the optimal solution to $\operatorname{SYSTEM}(U, A, C)$ if and only if there exists a Lagrangian vector $\mu^{*} \geq 0$ such that for every user $r \in R$ we have

$$
x_{r}^{*}=D_{r}\left(\lambda_{r}\right)
$$

where

$$
\lambda_{r}=\sum_{j \in R} \mu_{j}^{*}
$$

and for every link $j \in J$ we have

$$
\begin{gathered}
\sum_{r \in H(j)} x_{r}^{*} \leq C_{j} \\
\mu_{j}^{*}=0, \quad \text { if }\left(C_{j}-\sum_{r \in H(j)} D_{r}\left(\lambda_{i}\right)\right)<C_{j} .
\end{gathered}
$$

\section{Iterative Algorithm}

While the problem $N E T W O R K(A, C ; w)$ is tractable, it would be difficult to implement a solution in any centralized manner and Kelly et al. propose instead the use of a decentralized (dual) algorithm that iteratively computes an optimal solution. Adapt to the framework considered here, the algorithm is given as follows.

At iteration step $k$, each user knows the price

$$
\lambda_{r}^{k-1}=\sum_{j \in r} \mu_{j}^{k-1}
$$

that user $r$ was charged in the previous step $k-1$ under the link price vector $\mu^{k-1}=\left(\mu_{j}^{k-1}, j \in J\right)$. User $r$ then chooses the transmission rate $x_{r}^{k}$ given by

$$
x_{r}^{k}=D_{r}\left(\lambda_{r}^{k-1}\right),
$$

and the network updates its link prices by setting

$$
\mu_{j}^{k}=\left[\mu_{j}^{k-1}+\alpha_{j}\left(\sum_{r \in H(j)} x_{r}^{k}-C_{j}\right)\right]^{+},
$$

where $\alpha_{j}>0(j \in J)$ is a small step size parameter.

Note that the above algorithm is identical to the iterative algorithm given in Section IV for the case where every user either only generates traffic (i.e., acts as a user $r \in R$ ) or relays traffic (i.e., acts a router/link $j \in J$ ). 


\section{Vi. Convergence Analysis}

In this section, we analyze the convergence behavior of the iterative algorithm of Section IV and characterize the properties of its limit points. As our main result, we show that the algorithm converges to a unique bandwidth allocation $x^{*}=\left(x_{n}^{*}, n \in \mathcal{N}\right)$ that maximizes the sum of the users' utilities.

\section{A. Convergence of the Price Vector $\mu^{k}$}

We first show that

$$
\lim _{k \rightarrow \infty}\left\|\mu^{k}-\mu^{k+1}\right\|=0 .
$$

Let the Lyapunov function $\Phi: \Re_{+}^{N} \rightarrow \Re_{+}$be defined as

$$
\Phi(\mu)=\sum_{n \in \mathcal{N}} \int^{\mu_{n}+\lambda_{n}} D_{n}(\zeta) d \zeta-\sum_{n \in \mathcal{N}} \mu_{n} C_{n},
$$

where

$$
\lambda_{n}=\sum_{m \in r_{n}} \mu_{m} .
$$

We note that the above expression is of the same form as the Lyapunov function used in [3] to analyze the algorithm of Subsection V-D. We have the following result.

Lemma 6: There exists a constant $L>0$ such that

$$
\|\nabla \Phi(\mu)-\nabla \Phi(\eta)\| \leq L\|\mu-\eta\|, \quad \mu, \eta \in \Re_{+}^{N} .
$$

By the same argument as used by Kelly et al. in [3] to prove convergence for the algorithm of Subsection V-D, we then obtain the following result.

Proposition 2: When the step size parameters are such that

$$
1-\frac{\alpha_{n} L}{2}>0, \quad n \in \mathcal{N},
$$

then we have that

$$
\lim _{k \rightarrow \infty}\left\|\mu^{k}-\mu^{k+1}\right\|=0 .
$$

Note that the above proposition does not imply that the sequence of vectors $\left(\mu^{k}, k \geq 1\right)$ converges to an equilibrium price vector $\mu^{*}$.

\section{B. Properties of Limit Points}

Let $e_{n}^{k}=\left(x_{n}^{k}, y_{n}^{k}, \mu_{n}^{k}\right)$ be the allocation by user $n$ at iteration step $k$, let $e^{k}=\left(e_{n}^{k}, n \in \mathcal{N}\right)$ be the allocation vector at step $k$, and let $E=\left(e^{k}, k \geq 1\right)$ be the allocation sequence generated by the iterative algorithm. In this section, we show that every limit point $e^{*}$ leads to an allocation which maximizes the net benefit of each user. We first derive a few preliminary lemmas.

The next lemma states that sequence given by $E$ is bounded.

Lemma 7: There exists a constant $B>0$ such that

$$
\left\|e^{k}\right\|<B, \quad k \geq 1 \text {. }
$$

Using the above lemma, we obtain the following result (see [8]).
Lemma 8: Every infinite subset of $E$ has a limit point.

For every user $n \in \mathcal{N}$, we define the function $f_{n}(e)$ by

$$
\begin{aligned}
f_{n}(e)= & \left(x_{n}-D_{n}\left(\mu_{n}+\lambda_{n}\right)\right)^{2} \\
& +\left(y_{n}-I_{n}\left(\mu_{n}, \mu_{-n}\right)\right)^{2} \\
& +\mu_{n}\left(C_{n}-x_{n}-y_{n}\right),
\end{aligned}
$$

where $e=\left(e_{n}, n \in \mathcal{N}\right)$ with $e_{n}=\left(x_{n}, y_{n}, \mu_{n}\right) \in \Re_{+}^{3}$. The next lemma establishes that for every user $n \in \mathcal{N}$, the sequence $\left(f_{n}\left(e^{k}\right), k \geq 1\right)$ converges to 0 .

Lemma 9: We have

$$
\lim _{k \rightarrow \infty} f_{n}\left(e^{k}\right)=0 .
$$

Proving the above lemma is non-trivial; we refer to [5] for a derivation. The next proposition characterizes the limit points of $E$.

Proposition 3: Let $e^{*}$ be a limit point of the sequence $E=$ $\left\{e^{k}, k \geq 1\right\}$. For every user $n \in \mathcal{N}$, we then have that

$$
\begin{aligned}
x_{n}^{*} & =D_{n}\left(\mu_{n}^{*}+\lambda_{n}^{*}\right) \\
y_{n}^{*} & =I_{n}\left(\mu_{n}^{*}, \mu_{-n}^{*}\right) \\
\mu_{n}^{*} & =0, \quad \text { if } x_{n}^{*}+y_{n}^{*}<C_{n} .
\end{aligned}
$$

Proof: Note that the function $f_{n}(e)$ is continuous in $e$ for every user $n \in \mathcal{N}$. Combining this fact with Lemma 9, it then follows immediately that

$$
f_{n}\left(e^{*}\right)=0, \quad n \in \mathcal{N},
$$

or

$$
\begin{gathered}
\left(x_{n}-D_{n}\left(\mu_{n}+\lambda_{n}\right)\right)^{2}+\left(y_{n}-I_{n}\left(\mu_{n}, \mu_{-n}\right)\right)^{2}+\ldots \\
+\mu_{n}\left(C_{n}-x_{n}-y_{n}\right)=0, \quad n \in \mathcal{N} .
\end{gathered}
$$

Proposition 3 then follows immediately from the above equation.

Combining Proposition 3 with our discussion in Section III, it follows that at every limit point $e^{*}$ the allocation $\left(x_{n}^{*}, y_{n}^{*}, \mu_{n}^{*}\right)$ maximizes the net benefit of user $n$.

\section{System Properties}

In the subsection, we show that the algorithm converges to a unique bandwidth allocation which maximizes the sum of the utilities of all users.

Consider the (social welfare) maximization problem

$$
\max \sum_{n \in \mathcal{N}} U_{n}\left(x_{n}\right)
$$

$$
\begin{aligned}
\text { subject to } & A x \leq C \\
\text { over } & x \geq 0,
\end{aligned}
$$

where $A$ is the routing matrix that we defined in Section II and $C=\left(C_{n}, n \in \mathcal{N}\right)$ is the capacity vector. 
Note that this maximization problem is of the same form as the system problem considered by Kelly et al. (see Section V). Furthermore, it can be shown that the properties of a limit point $e^{*}$ as given in Proposition 3 are equivalent to the properties of Proposition 1 for the system problem considered in Section V. Combining these two observations, we obtain the following result.

Proposition 4: Let $x^{*}=\left(x_{n}^{*}, n \in \mathcal{N}\right)$ be the bandwidth allocation at a limit point of the sequence $E=\left(e^{k}, k \geq 1\right)$. Then $x^{*}$ is the unique optimal solution to (3).

Proof: We relate revenue optimization problem of each node in our work with the user optimization problem $U S E R\left[U_{n} ; \lambda_{n}\right]$ in [3]. There is a difference between the two scenarios. In our work, a node is charged for using transmission bandwidth of related nodes, while in $U S E R\left[U_{n} ; \lambda_{n}\right]$ a user is charged for using wired network links. We regard the bandwidth of node as a virtual link connecting any two nodes as in [3]. Use of such virtual links is charged.

By Proposition 3, we have that for each node $n \in \mathcal{N}$, if $\left(x_{n}^{*}, y_{n}^{*}, \mu_{n}^{*}\right)$ is a limit point of the sequence, $\left(x_{n}^{k}, y_{n}^{k}, \mu_{n}^{k}\right) k \in$ $\mathbb{N}$, it has the following properties:

$$
\begin{gathered}
x_{n}^{*}=D_{n}\left(\sum_{j=1}^{m} \mu_{r_{n_{j}}}\right) \\
\sum_{m \in H(n)} x_{m}^{*} \leq C_{n} \\
\mu_{n}=0, \text { if } \sum_{m \in H(n)} x_{m}^{*}<C_{n} .
\end{gathered}
$$

These properties show that each node actually pays every node along its route, including the node itself. This makes the node revenue optimization problem identical to the user optimization problem $U S E R\left[U_{n} ; \lambda_{n}\right]$ in [3].

Let $x^{*}=\left(x_{1}^{*}, \cdots, x_{N}^{*}\right)$ be the limit point of node transmission rate vector. Due to the constraint $A x \leq C$, we have that $x_{n} \leq C_{n}$ for any node $n$. Then our assumption of the utility function in Assumption 1 is the same as Assumption 2. Based on the above properties of the vector $x^{*}$, we apply Proposition 1 and obtain that $x^{*}$ is the unique limit point of node transmission rate vector $x$. Furthermore, the limit point $x^{*}$ solves the system maximization problem $S Y S T E M[U, A, C]$, thus maximizing the network social welfare.

The next corollary then follows immediately from Proposition 4.

Corollary 1: The sequence $\left(x^{k}, k \geq 1\right)$ converges to the unique bandwidth allocation $x^{*}=\left(x_{1}^{*}, \cdots, x_{N}^{*}\right)$ which solves (3).

Note that the above analysis only establishes that the algorithm converges to a unique bandwidth allocation; this does not imply it converges to a unique price vector $\mu^{*}$. Indeed, it can be shown the system equilibrium $e^{*}$ may not be unique (see [5] for an example).

\section{NUMERICAL RESUlTS}

In this section, we illustrate the iterative algorithm of Section IV and the results of Section VI through a numerical case study.

We consider a network consisting of 25 users (nodes). Figure 3 illustrates the network topology in terms of the routes of individual flows. The bandwidth capacity of every user is set equal to 10 packets/second. In addition, we assume that each user has the same utility function given by

$$
U(x)=\left\{\begin{array}{ll}
\lg (x+1) & x \in[0, C] \\
\lg (C+1) & x \in(C,+\infty)
\end{array} .\right.
$$

Setting the step size parameter $\alpha_{n}$ equal to 0.0007 for every user $n \in \mathcal{N}$, we simulated the iterative algorithm of Section IV for 800 iteration steps.

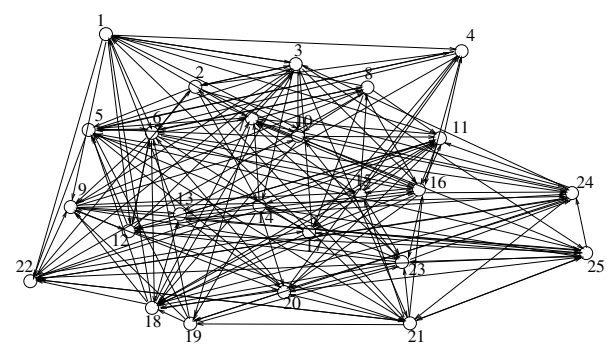

Fig. 3. Network Traffic Flows

Figure 4, and Figure 5, indicate the trajectories of the transmission rates $x_{n}$, and the price $\mu_{n}$, respectively, at individual user $n \in \mathcal{N}$. We note that the system converges to an equilibrium rate allocation, and an equilibrium price vector, within 600 iterations. The equilibrium transmission rates and prices are given in Table I. Note that the equilibrium price $\mu_{n}^{*}$ is equal to 0 at users that do not fully use their transmission capacity, i.e., if $x_{n}^{*}+y_{n}^{*}<10$.

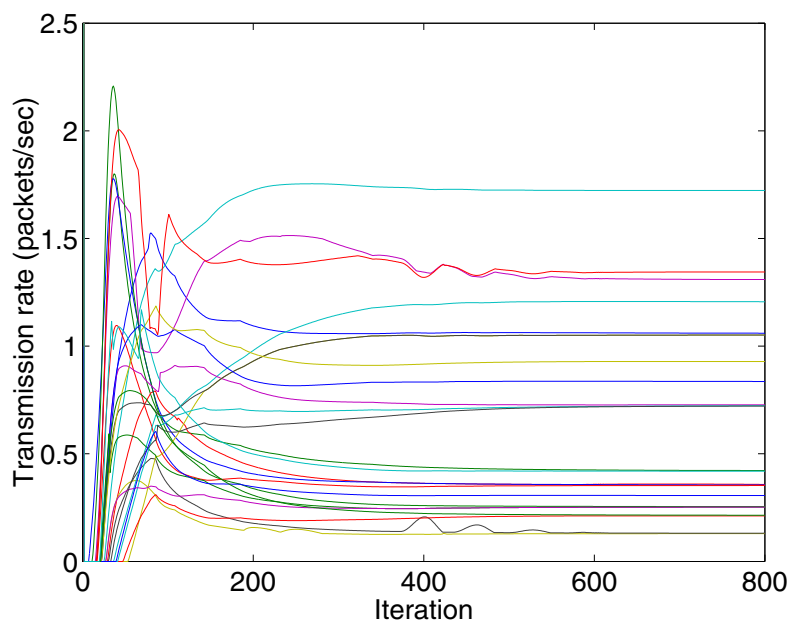

Fig. 4. Trajectories of the transmission rates $x_{n}$.

We run the simulation using different sets of initial values for the transmission rates and prices, and found that the system always converged to the same equilibrium rate and 


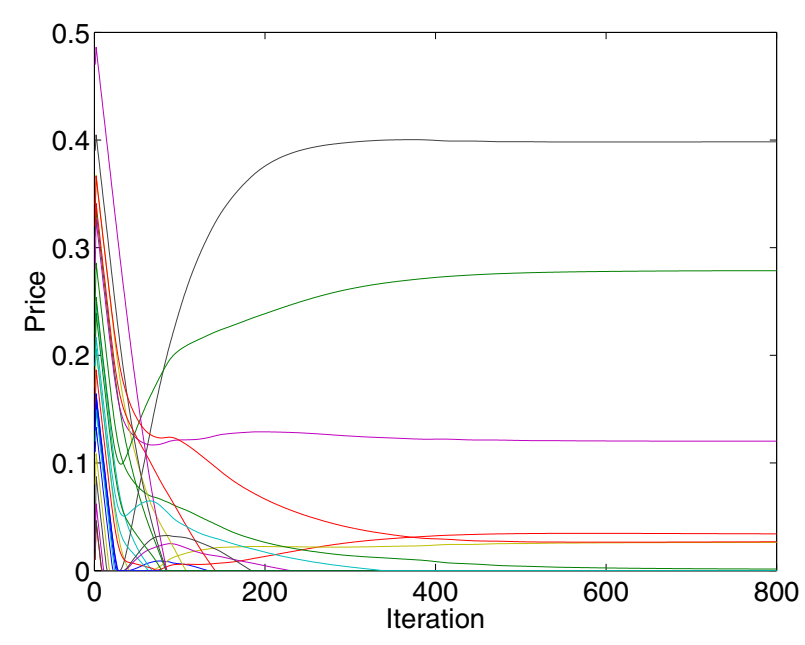

Fig. 5. Trajectories of the price $\mu_{n}$.

TABLE I

EQUILIBRIUM TRANSMISSION RATES AND PRICES

\begin{tabular}{|c|c|c|c|}
\hline User & $x_{n}^{*}$ & $y_{n}^{*}$ & $\mu_{n}^{*}$ \\
\hline 1 & 1.0601 & 7.9827 & 0 \\
\hline 2 & 0.4223 & 6.2287 & 0 \\
\hline 3 & 0.3574 & 9.6426 & 0.0342 \\
\hline 4 & 1.7232 & 6.5354 & 0 \\
\hline 5 & 1.3095 & 8.6905 & 0.1202 \\
\hline 6 & 0.1290 & 9.8710 & 0.0262 \\
\hline 7 & 0.1314 & 9.8686 & 0.3983 \\
\hline 8 & 0.3553 & 4.0663 & 0 \\
\hline 9 & 0.2545 & 9.7455 & 0.0015 \\
\hline 10 & 0.3526 & 6.0685 & 0 \\
\hline 11 & 1.2062 & 7.0717 & 0 \\
\hline 12 & 0.7275 & 8.9532 & 0 \\
\hline 13 & 1.0516 & 6.1872 & 0 \\
\hline 14 & 1.0516 & 5.8930 & 0 \\
\hline 15 & 0.8358 & 6.0169 & 0 \\
\hline 16 & 0.2522 & 9.7478 & 0.2786 \\
\hline 17 & 0.2116 & 7.4388 & 0 \\
\hline 18 & 0.7231 & 8.9465 & 0 \\
\hline 19 & 0.2522 & 4.5585 & 0 \\
\hline 20 & 0.9286 & 6.6787 & 0 \\
\hline 21 & 0.7214 & 7.8929 & 0 \\
\hline 22 & 0.3063 & 7.6517 & 0 \\
\hline 23 & 0.2146 & 5.3223 & 0 \\
\hline 24 & 1.3445 & 8.6555 & 0.0268 \\
\hline 25 & 0.4193 & 9.2796 & 0 \\
\hline & & & \\
\hline
\end{tabular}

price vector. This result might indicate that large-scale ad hoc networks typically have a unique system equilibrium $e^{*}=\left(x^{*}, y^{*}, \mu^{*}\right)$.

\section{EXTENSIONS}

In our discussion so far, we ignored the fact the users in a wireless ad hoc network may have limited battery resources. In this section, we outline how this effect can be incorporated into our model. For this extension, we will assume that each user transmits at a fixed power level and each packet transmission attempt drains the battery by the same amount.

\section{A. Battery Cost}

When the battery of an individual users is low, then this user will be reluctant to relay packets for other users as this might impact the user's ability to send its own traffic. We model this effect by associating a battery cost $p_{n}$ with each user $n \in \mathcal{N}$, where $p_{n}$ is higher the lower the battery level at user $n$. Roughly, $p_{n}$ captures the potential cost incurred if user $n$ uses its battery to send data now and this energy is not available at a future time to send its own data that might have a high value to user $n$, or to relay for other users at a high price $\mu_{n}$. We assume that each user $n$ decides on its battery cost $p_{n}$ as a function of its current battery level. Furthermore, we assume that the cost $p_{n}$ changes slowly compared with the time-scale at which users update their bandwidth allocation $\left(x_{n}, y_{n}\right)$ and the price $\mu_{n}$; this assumption will allow us to treat $p_{n}$ as a constant.

Let $p_{n}$ be the battery cost of user $n$, then we associate the following maximization problem with user $n$,

$$
\max _{x_{n}, y_{n}, \mu_{n}}\left\{U_{n}\left(x_{n}\right)-x_{n} \lambda_{n}+y_{n} \mu_{n}-p_{n}\left(x_{n}+y_{n}\right)\right\} .
$$

$$
\begin{array}{ll}
\text { subject to } & x_{n}+y_{n} \leq C_{n} \\
& y_{n} \leq I_{n}\left(\mu_{n}, \mu_{-n}\right) \\
& x_{n}, y_{n}, \mu_{n} \geq 0 .
\end{array}
$$

Note that this problem is similar to the maximization problem $U S E R\left(U_{n}, \mu_{-n}\right)$, however the above problem also accounts for the cost for draining the battery when user $n$ transmits at rate $x_{n}+y_{n}$.

In this case, a similar analysis as given in Section IV leads to the following update algorithm given by Figure 6 . Furthermore, one can show that for this iterative algorithm

\section{Bandwidth Allocation:}

If $D_{n}\left(\mu_{n}^{k-1}+\lambda_{n}^{k-1}+p_{n}\right)+i_{n}^{k-1} \geq C_{n}$, then set

$$
\begin{aligned}
& x_{n}^{k}=D_{n}\left(\mu_{n}^{k-1}+\lambda_{n}^{k-1}+p_{n}\right) \\
& y_{n}^{k}=C_{n}-x_{n}^{k} ;
\end{aligned}
$$

else if $D_{n}\left(\lambda_{n}^{k-1}+p_{n}\right)+i_{n}^{k-1}<C_{n}$, then set

$$
\begin{aligned}
& x_{n}^{k}=D_{n}\left(\lambda_{n}^{k-1}+p_{n}\right) \\
& y_{n}^{k}=i_{n}^{k-1} ;
\end{aligned}
$$

else set

$$
\begin{aligned}
& x_{n}^{k}=C_{n}-i_{n}^{k-1} \\
& y_{n}^{k}=i_{n}^{k-1} ;
\end{aligned}
$$

Price Update:

$\mu_{n}^{k}=p_{n}+\left[\mu_{n}^{k-1}+\alpha_{n}\left(D_{n}\left(\mu_{n}^{k-1}+\lambda_{n}^{k-1}+p_{n}\right)+i_{n}^{k-1}-C_{n}\right)\right]^{+}$.

Fig. 6. Iterative algorithm with battery cost $p_{n}$.

the system will converge to a bandwidth allocation which maximizes the following objective function

$$
\max \sum_{n \in \mathcal{N}}\left(U_{r}\left(x_{n}\right)-x_{n}\left(p_{n}+\sum_{m \in r_{n}} p_{m}\right)\right)
$$




$$
\begin{array}{ll}
\text { subject to } & x_{n}+\sum_{m \in G(n)} x_{m} \leq C_{n}, \quad n \in \mathcal{N}, \\
& x_{n} \geq 0, \quad n \in \mathcal{N} .
\end{array}
$$

Note that this implies that a user will reduce its transmission rate if (a) its own battery is low or (b) its route passes through another user with a low battery.

\section{B. Interference Cost}

Interference between users in an ad hoc network can cause bit errors and packet loss. In the case of loss, data packets will have to be retransmitted, thus further depleting and incurring an additional interference cost. Here, we model this interference cost for a ad hoc network which consists of a set $S$ of overlapping cells. We identify a cell $s \in S$ by the set of users belonging to $s$. We assume that each cell is assigned communication channel (i.e., frequency, spreadspectrum code) such that there is no interference between data transmissions in different cells. Let $C_{s}$ be the capacity of the communication channel of cell $s$. Users located within the same cell $s \in S$ communicate with others by broadcasting packets on this channel. Users which belong to more than one cell can be used to relay packets between cells and ensure that each user can reach every other user in the network. When two or more nodes access the channel in cell $s$ at the same time, then their packets will collide and are lost. Lost packets are transmitted at a later time (see contention-based multiaccess protocols such as slotted Aloha and CSMA/CA [7]).

We use the following notation. Let $H(s)$ be the set of users whose route passes through cell $s \in S$. Furthermore, let $R(n)$ be the set of cells to which user $n$ relays packets, and let $s_{n}$ be the cell in which user $n$ send its own traffic. Let $x_{n}$ be the transmission rate of user $n$ and let $y_{n, s}$ be the transmission rate that user $n$ allocates to relay packets to other nodes in cell $s$. The total transmission rate that user $n$ allocates to relay packets is then given by $y_{n}=\sum_{s \in R(n)} y_{n, s}$. We assume users which belong to more than one cell can only access one channel at each time instant, i.e., we have that the constraint that $x_{n}+y_{n} \leq C_{n}$, where $C_{n}$ is the transmission capacity of user $n$. Let $q_{n, s}$ be the expected number of times user $n$ has to transmit a packet in cell $s$ to get it successfully delivered. Note that $q_{n, s}$ depends on the level of interference in cell $s$, i.e., we have $q_{n, s}=1 /\left(1-P_{n, s}\right)$ where $P_{n, s}$ is the probability that a transmission attempt of user $n$ experiences interference and is lost. When many users share the same channel, then probability $P_{n, s}$ will be identical for all users sharing the channel $s$ and only depends on the aggregated rate of transmission attempts on this channel. Let $P_{s}\left(z_{s}\right)$ be the probability that a transmission attempt experiences a collision and formulated as a function of the transmission rate

$$
z_{s}=\sum_{n \in s}\left(x_{n}+y_{n, s}\right)
$$

in cell $s$. We make the following assumption.
Assumption 3: The function $P_{s}\left(z_{s}\right), s \in S$, is continuous and we have that $P_{s}(0)=0$ and

$$
\lim _{z_{s} \rightarrow \infty} P_{s}\left(z_{s}\right)=1 \text {. }
$$

Let $p_{n}$ be the battery cost for one transmission attempt at user $n$. The total battery cost for forwarding a packet at a node $n \in s$ is then equal to

$$
p_{n} q_{s}\left(z_{s}\right)=p_{n} \frac{1}{1-P_{s}\left(z_{s}\right)} .
$$

To simplify the analysis, we assume that the battery costs of all users in the same cell $s$ are identical and equal to $p_{s}$. The total battery cost for forwarding a packet at a node $n \in s$ is then equal to $p_{s} q_{s}\left(z_{s}\right)$.

We then associate with each user $n$ the following maximization problem.

$$
\begin{aligned}
\max _{x_{n},\left(y_{n, s}: s \in R(n)\right),\left(\mu_{n, s}: s \in R(n)\right)} & \left\{U_{n}\left(x_{n}\right)+\right. \\
+ & \sum_{s \in R(n)} y_{n, s}\left(\mu_{n, s}-p_{s} q_{s}\left(z_{s}\right)\right)- \\
- & \left.x_{n}\left(\lambda_{n}+p_{s_{n}} q_{s_{n}}\left(z_{s_{n}}\right)\right)\right\}
\end{aligned}
$$

where $\mu_{n, s}$ is the price that user $n$ charges for forwarding packets to cell $s$. Note that in the above maximization problem, user $n$ is allowed to charge a different price $\mu_{n, s}$ for relaying traffic to different cells $s \in S$. This accounts for the fact that user $n$ wants to charge a higher price for traffic to be transmitted in a cell $s$ with a high channel access cost $p_{s} q_{s}\left(z_{s}\right)$.

The same argument as given in Section IV leads to the following update algorithm given by Figure 7 .

\section{Bandwidth Allocation:}

If $D_{n}\left(\mu_{n}^{k-1}+\lambda_{n}^{k-1}+p_{s_{n}} q_{s_{n}}\left(z_{s_{n}}^{k-1}\right)\right)+i_{n}^{k-1} \geq C_{n}$, then set $x_{n}^{k}=D_{n}\left(\mu_{n}^{k-1}+\lambda_{n}^{k-1}+p_{s_{n}} q_{s_{n}}\left(z_{s_{n}}^{k-1}\right)\right)$ $y_{n}^{k}=C_{n}-x_{n}^{k}$;

else if $D_{n}\left(\lambda_{n}^{k-1}+p_{s_{n}} q_{s_{n}}\left(z_{s_{n}}^{k-1}\right)\right)+i_{n}^{k-1}<C_{n}$, then set $x_{n}^{k}=D_{n}\left(\lambda_{n}^{k-1}+p_{s_{n}} q_{s_{n}}\left(z_{s_{n}}^{k-1}\right)\right)$ $y_{n}^{k}=i_{n}^{k-1}$

else set

$$
\begin{aligned}
& x_{n}^{k}=C_{n}-i_{n}^{k-1} \\
& y_{n}^{k}=i_{n}^{k-1}
\end{aligned}
$$

Price Update:

$$
\begin{aligned}
& \mu_{n}^{k}= p_{s} q_{s}\left(z_{s}^{k-1}\right)+ \\
&+\left[\mu_{n}^{k-1}+\alpha_{n}\left(D_{n}\left(\mu_{n}^{k-1}+\lambda_{n}^{k-1}+p_{s} q_{s}\left(z_{s}^{k-1}\right)\right)+\right.\right. \\
&\left.\left.\quad+i_{n}^{k-1}-C_{n}\right)\right]^{+}, \quad s \in R(n) .
\end{aligned}
$$

Fig. 7. Iterative algorithm with interference cost $p_{s} q_{s}\left(z_{s}\right)$.

Furthermore, the same argument as used in Section IV can be used to show that the above system converges to an equilibrium bandwidth allocation which approximates the solution to the following maximization problem,

$$
\max \sum_{n \in \mathcal{N}} U_{n}\left(x_{n}\right) \text {. }
$$




$$
\begin{array}{ll}
\text { subject to } & x_{n}+\sum_{m \in G(n)} x_{m} \leq C_{n}, \quad n \in \mathcal{N}, \\
& \sum_{n \in H(s)} x_{s} \leq C_{s}, \quad s \in S \\
& x_{n} \geq 0, \quad n \in \mathcal{N} .
\end{array}
$$

The same argument as given in [3] shows that the approximation will become better as the functions $P_{s}\left(z_{s}\right), s \in S$, approach the step function

$$
\hat{P}(z)= \begin{cases}0 & z<C_{s}, \\ 1 & z \geq C_{s} .\end{cases}
$$

\section{COnclusions}

In this paper, we use pricing to give an incentive to network users for sharing their bandwidth in ad hoc networks. We define the pricing model and then propose the adaptation algorithm for users to optimize their net benefit. Moreover, we show that the algorithm converges to the unique bandwidth allocation which maximizes the sum of the users' utility (social welfare).

For our analysis, we focused on the case where the demand is very elastic. Characterizing the system equilibrium for the general case is still an open problem.

\section{REFERENCES}

[1] L. Blažević, L. Buttyán, S. Čapkun, S. Giordano, J.-P. Hubaux, and J.-Y. Le Boudec, "Self-organizing in mobile ad hoc networks: the approach of terminodes," IEEE Communications Magazine, vol. 39, no. 6, pp. 166-174, June 2001.

[2] L. Buttyán and J.-P. Hubaux, "Nuglets: a virtual currency to stimulate cooperation in self-organizing mobile ad hoc networks," Tech. Rep. DCS/2001/001, Swiss Federal Institute of Technology, January 2001.

[3] F. Kelly, A. Maulloo, and D. Tan, "Rate control for communication networks: shadow prices, proportional fairness and stability," Journal of Operational Research Society, vol. 49, no. 3, pp. 237-252, March 1998.

[4] S. Shenker, "Fundamental design issues for the future internet," IEEE Journal on Selected Areas in Communication, vol. 13, no. 7, pp. 11761188, September 1995.

[5] Y. Qiu, "A market-based approach to bandwidth allocation for wireless ad hoc networks," M.Sc. thesis, University of Toronto, June 2002.

[6] D. Bertsekas, Nonlinear Programming, Athena Scientific, 1999.

[7] J. Kurose and K. Ross, Computer Networking: A Top-Down Approach Featuring the Internet, Addison Wesely, 2002.

[8] W. Rudin, Principles of Mathematical Analysis, NcGraw-Hill Inc., 1976. 